\title{
Acute Severe Mitral Regurgitation Secondary to Ischemic Papillary Muscle Rupture: A Case Report
}

\author{
Loba Alam ${ }^{1}$, Glenmore Lasam ${ }^{2}$, Robert Fishberg ${ }^{3}$, David Powell ${ }^{4}$ \\ 1. Internal Medicine, Overlook Medical Center, Summit, USA 2. Cardiology, Mt. Sinai Morningside, Summit, USA 3. \\ Cardiology, Overlook Medical Center, Summit, USA 4. Cardiology, Overlook Medical Center, Summit, USA
}

Corresponding author: Loba Alam, alamloba@gmail.com

\begin{abstract}
Mitral valve rupture secondary to ischemic papillary muscle necrosis is rare in the contemporary era due to improved revascularization techniques. However, when it does occur, prompt diagnosis and urgent surgical intervention can be lifesaving. A 69-year-old male with morbid obesity, hypothyroidism, and a family history of coronary artery disease presented to the hospital with chest pain and dyspnea that began five hours prior. He had an acute infero-postero-lateral myocardial infarction due to total occlusion of the left circumflex artery that was revascularized with the deployment of a drug-eluting stent. Two days after the myocardial infarction, the patient had an episode of ventricular tachycardia. He subsequently went into respiratory distress from flash pulmonary edema and developed cardiogenic shock due to acute mitral valve rupture. The patient underwent surgical mitral valve replacement, extracorporeal membranous oxygenation (ECMO), and hemodialysis. His course was complicated by an acute lower gastrointestinal bleed that progressed into multiorgan failure and eventually his demise. This case highlights the need to include papillary muscle rupture high on the differential when evaluating a hemodynamically unstable patient in the setting of an acute myocardial infarction (MI). Rapid diagnosis by urgent bedside echocardiogram and surgical intervention is crucial.
\end{abstract}

Review began 03/07/2021 Review ended 03/15/2021 Published 03/19/2021

๑) Copyright 2021 Alam et al. This is an open access article distributed under the terms of the Creative Commons Attribution License CC-BY 4.0., which permits unrestricted use, distribution, and reproduction in any medium, provided the original author and source are credited.
Categories: Cardiac/Thoracic/Vascular Surgery, Cardiology, Internal Medicine

Keywords: acute mitral regurgitation, papillary muscle rupture, transthoracic and transesophageal echocardiography, extracorporeal membranous oxygenation, mitral valve replacement

\section{Introduction}

Papillary muscle rupture is a rare and fatal complication of acute ST-segment elevation myocardial infarction in the contemporary age. Rupture of a papillary muscle leads to acute mitral valvular regurgitation with subsequent flash pulmonary edema and cardiogenic shock. A transesophageal echocardiogram can be used to explore the mitral valvular apparatus to understand the mechanism of mitral regurgitation. Prompt recognition and surgical intervention can be lifesaving, although surgical intervention carries high morbidity and mortality risk. We describe the case of an acute severe mitral regurgitation resulting from a posteromedial papillary muscle rupture which occurred within 48 hours of a myocardial infarction.

\section{Case Presentation}

A 69-year-old man with a history of morbid obesity, hypothyroidism, and a family history of coronary artery disease presented to the hospital with chest pain and dyspnea which began earlier on the morning of admission approximately five hours prior to hospital presentation. He had a normal cardiopulmonary physical exam and vital signs. Initial labs including hemogram and basic metabolic panel were normal. He had mild troponin elevation of $0.285 \mathrm{ng} / \mathrm{mL}$. He had normal vital signs. Initial ECG demonstrated ST-T wave changes in leads I, aVL, II, III, aVF and V1 - V6 suspicious for an acute infero-postero-lateral wall ST segment elevation myocardial infarction (STEMI) (Figure 1). 


\section{Cureus}

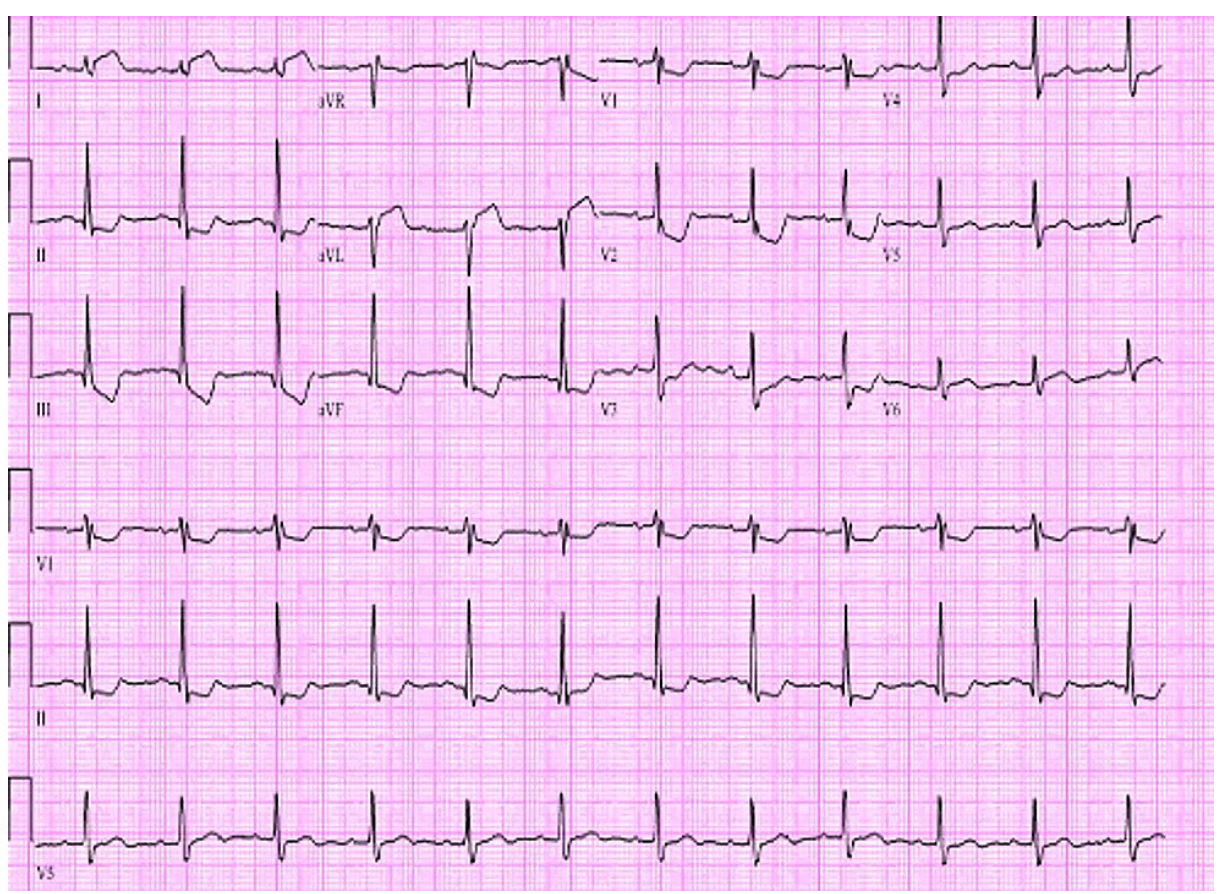

FIGURE 1: : ECG demonstrated ST-T wave changes in leads II, III, aVF and V1 - V6 suspicious for an acute inferior-posterior wall STEMI.

STEMI: ST segment elevation myocardial infarction

STEMI protocol was activated, and the patient was brought to the coronary catheterization laboratory emergently. A coronary angiogram revealed a left dominant circulation with a $100 \%$ occlusion of the proximal left circumflex artery. The lesion was acute and heavily thrombotic (Figure 2). 


\section{Cureus}

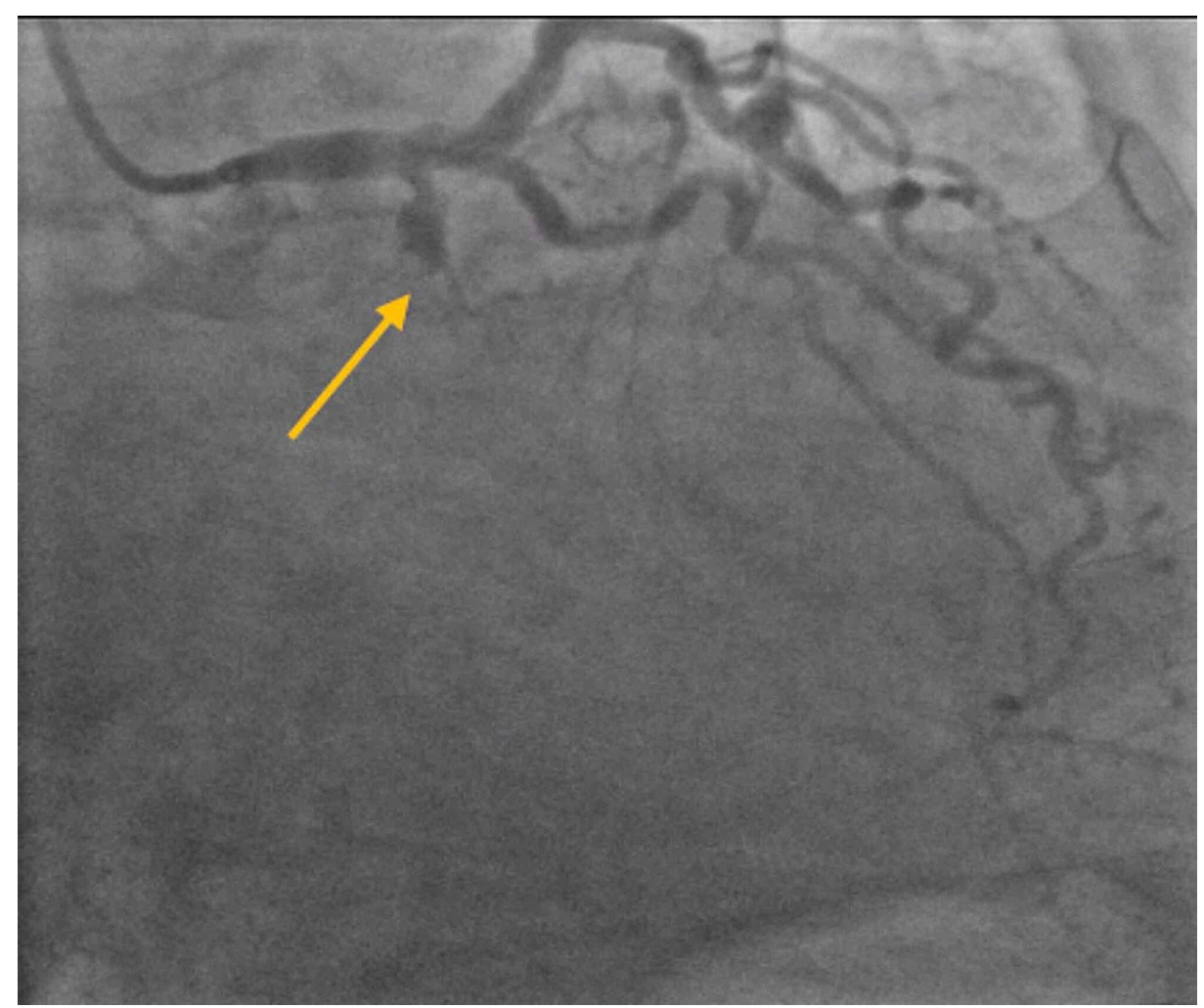

\section{FIGURE 2: Coronary angiogram revealed a left dominant circulation with a $100 \%$ occlusion of the proximal left circumflex artery (arrow).}

The proximal left circumflex lesion was successfully stented with a drug-eluting stent. The left ventriculogram revealed an estimated ejection fraction of $45 \%$ with hypokinesis of the inferior and inferolateral segments. The patient was started on aspirin and ticagrelor.

The patient was steadily improving until post-infarction day two when he developed sudden onset ventricular tachycardia requiring one synchronized cardioversion with conversion to sinus tachycardia. He subsequently developed respiratory distress, hypoxia with oxygen saturation of $85 \%$ on room air, and hypotension with blood pressure $80 \mathrm{~s} / 50 \mathrm{~s} \mathrm{mmHg}$, and sinus tachycardia with heart rate in the range of 130140 beats per minute. At this point, the differential diagnosis included ventricular free wall rupture, papillary muscle rupture, or recurrent myocardial infarction resulting in flash pulmonary edema and cardiogenic shock.

An urgent transthoracic echocardiogram (TTE) revealed acute mitral regurgitation (MR), the severity of which was difficult to assess due to tachycardia. Urgent left heart catheterization was performed to exclude acute stent closure. Coronary catheterization revealed a patent stent and otherwise unchanged coronary anatomy. In addition, right heart pressures were severely elevated, suggestive of acute MR and pulmonary edema. The right heart pressures were as follows: Mean right atrial pressure was $30 \mathrm{mmHg}$, the right ventricular pressure was $55 / 10 \mathrm{mmHg}$, the pulmonary artery pressure was $60 / 30 \mathrm{mmHg}$, and the pulmonary capillary wedge pressure was $45 \mathrm{mmHg}$ with a V-wave of $65 \mathrm{mmHg}$ (Figure 3). 


\section{Cureus}

3)

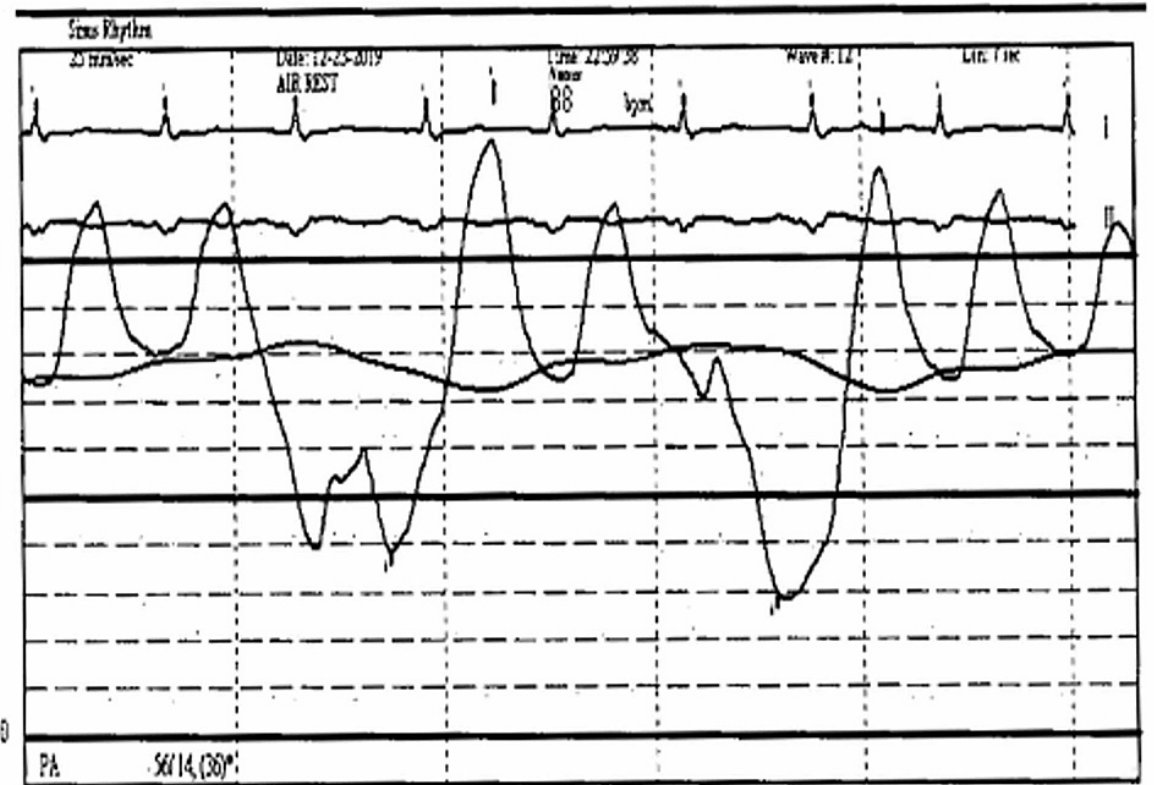

FIGURE 3: Coronary catheterization revealed severely elevated right heart pressures with pulmonary artery pressure was $60 / 30 \mathrm{mmHg}$ and a V-wave of $65 \mathrm{mmHg}$.

The cardiac output by the Fick method was 3.1 liters/minute with a cardiac index of $1.7 \mathrm{~L} / \mathrm{m} / \mathrm{m} 2$. After intraaortic balloon pump (IABP) insertion, the mean pressure was initially 80 to $85 \mathrm{mmHg}$. Intravenous nitroprusside was initiated, and the patient was intubated due to flash pulmonary edema causing acute hypoxic respiratory failure. Next, an urgent transesophageal echocardiogram (TEE) confirmed flail anterior mitral leaflet and chordae due to posteromedial papillary muscle rupture, as well as severe posteriorly directed mitral valve regurgitation (Figure 4). 


\section{Cureus}

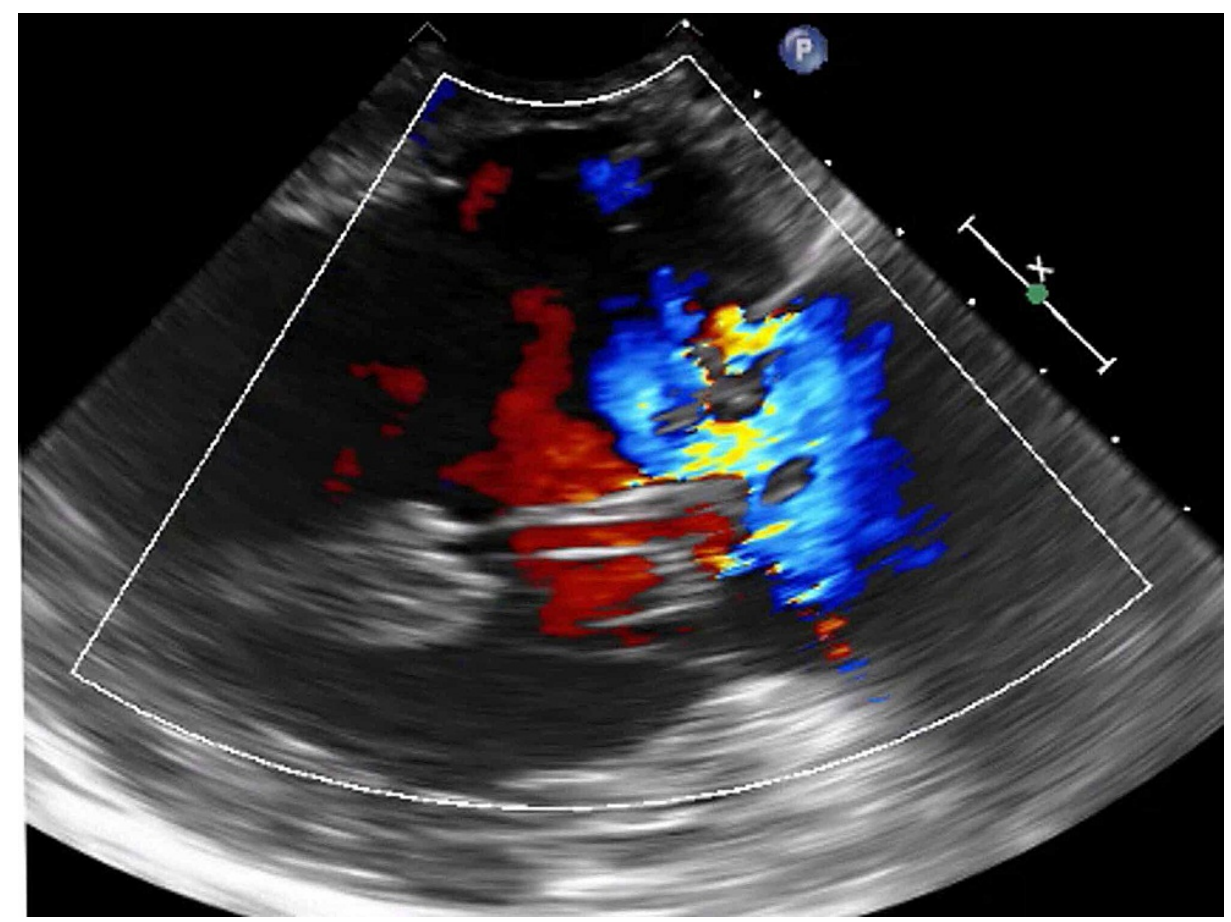

FIGURE 4: TEE confirmed flail anterior mitral leaflet and chordae due to posteromedial papillary muscle rupture, as well as severe posteriorly directed

TEE: Transesophageal Echocardiogram

The posteriorly directed MR, flail anterior mitral leaflet, and papillary muscle rupture can be further reviewed in supplemental videos (Videos 1- 5).

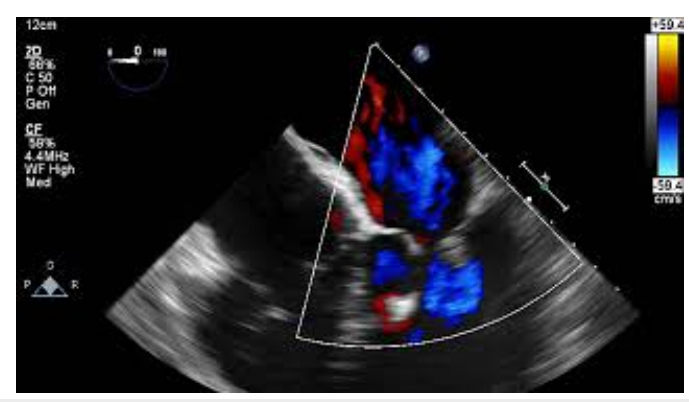

VIDEO 1: TEE Apical 4 chamber view with color demonstrating posterior directed mitral valve regurgitant flow.

TEE: Transesophageal Echocardiogram

View video here: https://youtu.be/D4FaacaJFVk

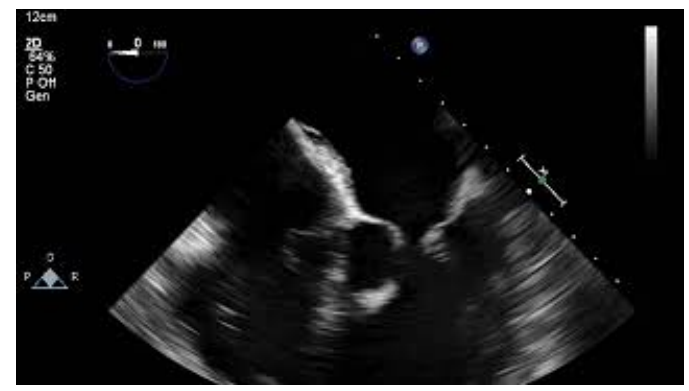




\section{Cureus}

VIDEO 2: TEE Apical 4 chamber view without color demonstrating flail motion of anterior mitral leaflet.

TEE: Transesophageal Echocardiogram

View video here: https://youtu.be/Do_hYTmCFIY

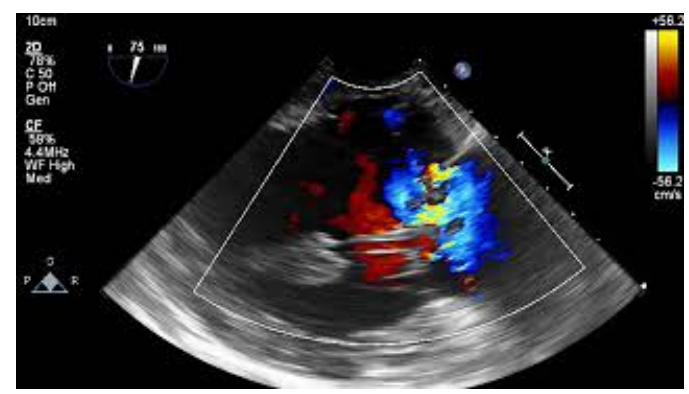

VIDEO 3: TEE Deep gastric parasternal long-axis view with color demonstrating mitral valve regurgitant flow.

TEE: Transesophageal Echocardiogram

View video here: https://youtu.be/sKPXIYG7Czo

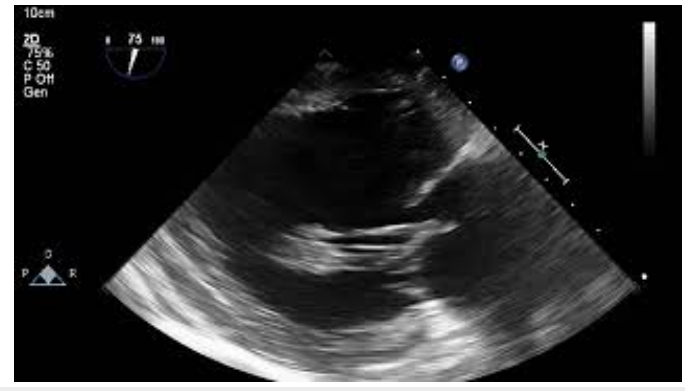

VIDEO 4: TEE Deep gastric parasternal long axis view without color demonstrating flail motion of anterior mitral leaflet with papillary muscle rupture.

TEE: Transesophageal Echocardiogram

View video here: https://youtu.be/Fy2qdmUhHoM

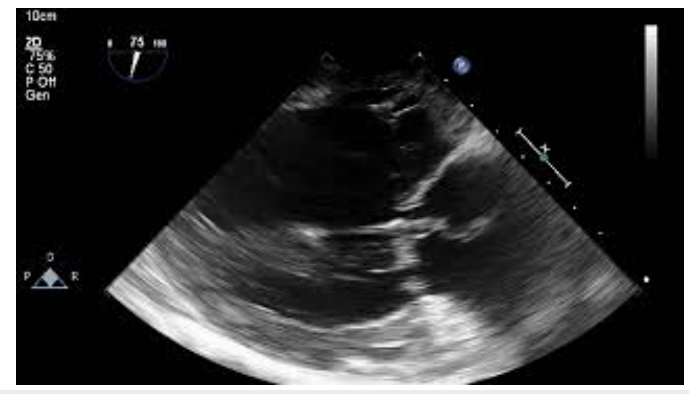

VIDEO 5: Apical 2 chamber view without color demonstrating flail motion of anterior mitral leaflet.

View video here: https://youtu.be/A_R5JgDnNwg

\section{Expert Consultation}

The patient remained hypotensive despite high dose inotropes and IABP. Cardiothoracic surgery was 


\section{Cureus}

consulted, and he was emergently taken to the operating room for venoarterial extracorporeal membranous oxygenation (ECMO) placement through a right femoral venoarterial route with percutaneous cannulation. In spite of the very high risk, the decision was made to perform open-heart surgery to replace the mitral valve.

\section{Final Diagnosis and Treatment}

Intraoperatively, the examination of the mitral valve confirmed rupture of the posterior papillary muscle attached to the cord of the anterior mitral leaflet (Figure 5).
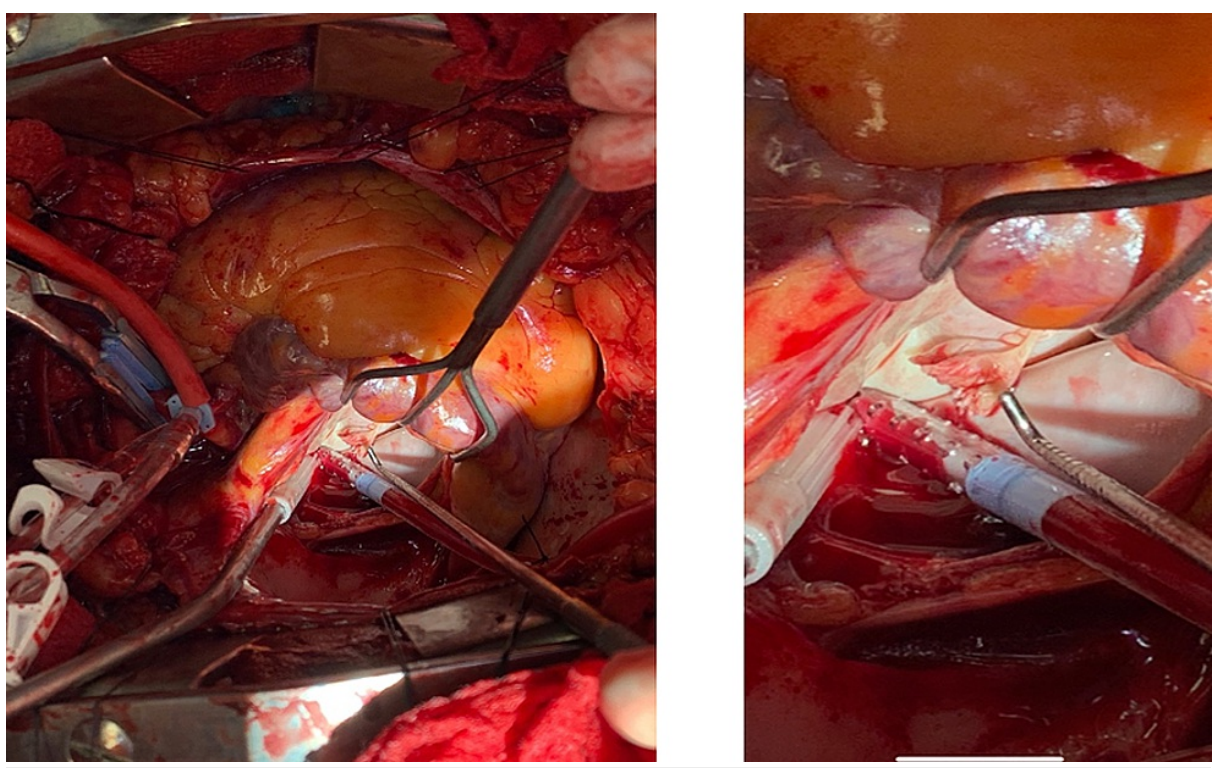

FIGURE 5: Intraoperatively, the examination of the mitral valve confirmed rupture of the posterior papillary muscle attached to the cord of the anterior mitral leaflet.

The mitral valve was excised and replaced by $33 \mathrm{~mm}$ Edwards Lifesciences pericardial tissue heart valve. The cardiopulmonary bypass duration was 147 minutes, after which the patient was once again connected to the ECMO circuit.

\section{Outcome and Follow Up}

Postoperatively, the patient required venoarterial ECMO support as well as hemodialysis for five days. Following that, although the patient made slow recovery, his course was complicated by acute lower gastrointestinal bleed that progressed to multiorgan failure. Despite aggressive treatment, the patient expired on postoperative day twelve.

\section{Discussion}

Acute MR caused by papillary muscle rupture is a rare and life-threatening complication of myocardial infarction. Rupture commonly occurs within five days of an infarct. During this period the cardiac muscle is weakened due to infarction and subsequent necrosis. Acute MR is a cardiac emergency as it presents with sudden onset flash pulmonary edema, hypotension, and cardiogenic shock. Fifty percent of patients with acute moderate to severe MR often have no audible murmur [1]. Severe MR may be silent because reduced ventricular function minimizes the atrioventricular gradient, regurgitant flow, and subsequent murmur [1]. Additionally, the acoustic transmission of the murmur may be obscured by obesity and respiratory distress.

This case highlights the appreciation of the mitral valve anatomy and its structural integrity. The anterolateral muscle is usually a single large trunk whereas the posteromedial muscle can have one to three heads [2]. Each papillary muscle sends chordae tendineae to attach to both the anterior and posterior mitral valve leaflets. Thus, rupture of either papillary muscle can result in flail of either leaflet. The anterolateral papillary muscle typically has dual blood supply from the left anterior descending and left circumflex arteries. The posteromedial papillary muscle has a singular blood supply from the posterior descending artery which is a branch of the right coronary artery in a right dominant system or the left circumflex artery in a left dominant system. Thus, owing to its singular arterial blood supply, the posteromedial muscle is more susceptible to infarction and subsequent rupture [3]. 
TTE is often the initial modality used to diagnose papillary muscle rupture and it offers a sensitivity of $65 \%$ to $85 \%$ [4]. However, the mitral valve is a posterior structure, thus TEE offers superior visibility and diagnostic sensitivity of $95 \%$ to $100 \%$ [5]. The use of color flow Doppler to measure eccentric regurgitant jets improves diagnostic sensitivity in both types of echocardiogram modalities [6]. Once papillary muscle rupture is established, treatment includes emergent surgical intervention with mitral valve repair if the necrosis is limited or prosthetic valvular replacement if the tissue is too friable to be repaired. Concurrent coronary artery bypass graft should be considered as it has shown to improve early and long-term survival $[7]$.

While in-hospital mortality rates of surgical correction of acute MR secondary to papillary muscle rupture approach 90\% [8], emergent surgical valvular correction approaches a mortality rate of 40\% [9]. Low cardiac output states that require ECMO and hemodialysis post-procedure are associated with the poorest outcome [10]. Thus, in the modern era of percutaneous interventions for structural heart disease, an alternate approach to surgery such as transcatheter mitral valve repair could be considered. Indeed, there are isolated case reports where percutaneous MitraClip has been performed to treat acute severe MR in the setting of papillary muscle rupture and cardiogenic shock [11].

\section{Conclusions}

Although with early revascularizations and improved techniques, papillary muscle rupture has become a rare mechanical complication of acute myocardial infarction, physicians should have papillary muscle rupture in the differential when evaluating hemodynamically unstable patients in the setting of acute myocardial infarction. Rapid diagnosis and treatment can be lifesaving.

\section{Learning Objectives}

1. Papillary muscle rupture should be considered in patients with acute decompensation after acute myocardial infarction.

2. The posteromedial muscle is more susceptible to infarction and subsequent rupture due to its singular blood supply from the posterior descending artery.

3. Each papillary muscle sends chordae tendineae to attach to both the anterior and posterior mitral valve leaflets. Thus, rupture of either papillary muscle can result in flail of either leaflet.

4. Rapid diagnosis of papillary muscle rupture by coronary catheterization, TTE, and/or TEE is necessary in order for definitive treatment.

5. Definitive treatment includes traditional surgical repair/replacement, as well as contemporary percutaneous mitral valve repair.

\section{Additional Information \\ Disclosures}

Human subjects: Consent was obtained or waived by all participants in this study. Conflicts of interest: In compliance with the ICMJE uniform disclosure form, all authors declare the following: Payment/services info: All authors have declared that no financial support was received from any organization for the submitted work. Financial relationships: All authors have declared that they have no financial relationships at present or within the previous three years with any organizations that might have an interest in the submitted work. Other relationships: All authors have declared that there are no other relationships or activities that could appear to have influenced the submitted work.

\section{References}

1. Bursi F, Enriquez-Sarano M, Nkomo VT, Jacobsen SJ, Weston SA, Meverden RA, Roger VL: Heart failure and death after myocardial infarction in the community: the emerging role of mitral regurgitation. Circulation. 2005, 111:295.

2. Tempe DK, Datt V, Banerjee A, Gandhi A: Choardae tendineae from posteromedial papillary muscle inserting into the anterior mitral leaflet: an unusual presentation. Ann Card Anaesth. 2007, 10:63.

3. Fradley MG, Picard MH: Rupture of the posteromedial papillary muscle leading to partial flail of the anterior mitral leaflet. Circulation. 2011, 123:1044-104.

4. Czarnecki A, Thakrar A, Fang T, Lytwyn M, Ahmadie R, Pascoe E, Jassal DS: Acute severe mitral regurgitation: consideration of papillary muscle architecture. Cardiovasc Ultrasound. 2008, 6:5. 10.1186/1476-7120-6-5

5. Behar S, Reicher-Reiss H, Goldbourt U, Kaplinsky E: Comparison of accuracy of transesophageal versus transthoracic echocardiography for the detection of mitral valve prolapse with ruptured chordae tendineae (flail mitral leaflet). Am J Cardiol. 1991, 67:1251-5. 10.1016/0002-9149(91)90936-F

6. Antman EM, Morrow DA: Chapter 55 - ST-segment elevation myocardial infarction: management . Braunwald's heart disease - a textbook of cardiovascular medicine. Bonow RO (ed): Saunders, Philadelphia; 


\section{Cureus}

2011. 1150.

7. Schroeter T, Lehmann S, Misfeld M, Borger M, Subramanian S, Mohr FW, Bakthiary F: Clinical outcome after surgical correction of mitral regurgitation due to papillary muscle rupture. Circulation. 2008, 118:1528.

10.1016/j.athoracsur.2012.10.050

8. Nishimura RA, Gersh BJ, Schaff HV: The case for an aggressive surgical approach to papillary muscle rupture following myocardial infarction: "From paradise lost to paradise regained.”. Heart. 2000, 83:611-13. 10.1136/heart.83.6.611

9. Schroeter T, Lehmann S, Misfeld M, Borger M, Subramanian S, Mohr FW, Bakthiary F: Clinical outcome after mitral valve surgery due to ischemic papillary muscle rupture. Ann Thorac Surg. 2013, 95:820-824. 10.1016/j.athoracsur.2012.10.050

10. Obadia B, Théron A, Gariboldi V, Collart F: Extracorporeal membrane oxygenation as a bridge to surgery for ischemic papillary muscle rupture. Jour of Thor Car Sur. 2014, 147:82-84. 10.1016/j.jtcvs.2014.03.003

11. Valle JA, Miyasaka RL, Carroll JD: Acute mitral regurgitation secondary to papillary muscle tear. Is transcatheter edge-to-edge mitral valve repair a new paradigm?. Circ Cardiovasc Interv. 2017, 10:6. 10.1161/CIRCINTERVENTIONS.117.005050 